\title{
Um Símbolo no Domínio de Fresnel Para Utilização de Schmidl \& Cox em Esquemas OCDM
}

\author{
Vanderlan J. E. de Lima, Mateus de L. Filomeno, Ândrei Camponogara e Moisés V. Ribeiro
}

Resumo-Neste trabalho é apresentado um símbolo no domínio de Fresnel que possibilita a utilização das técnicas de sincronização temporal e na frequência propostas por Schmidl \& Cox em esquemas orthogonal chirp-division multiplexing. Nesse contexto, é demonstrado que um símbolo composto por duas metades idênticas no domínio de Fresnel possui duas metades idênticas no domínio do tempo, uma vez que as matrizes das transformadas discretas de Fresnel são circulantes.

Palavras-Chave-Multiplexação por divisão ortogonal em chirp, sincronização temporal, sincronização na frequência.

Abstract-This paper presents a symbol in the Fresnel domain that enables the use of the frequency and timing synchronization techniques proposed by Schmidl \& Cox in orthogonal chirp-division multiplexing schemes. In this context, it is demonstrated that a symbol composed of two identical halves in the Fresnel domain has two identical halves in the time domain, since the discrete Fresnel transform can be carried out by circulant matrices.

Keywords-Orthogonal chirp-division multiplexing, frequency synchronization, and timing synchronization.

\section{INTRODUÇÃO}

Nos últimos anos, esquemas orthogonal frequency-division multiplexing (OFDM) tem sido bastante explorados, principalmente pela elevada eficiência para lidar com canais seletivos em frequência. Recentemente, esquemas orthogonal chirp-division multiplexing (OCDM) foram propostos, tanto na banda base [1] como na banda passante [2], como uma alternativa ao convencional esquema OFDM, apresentando maior robustez à interferência intersimbólica.

Apesar das vantagens, alguns desafios devem ser superados a fim de utilizar esquemas OFDM ou OCDM, dentro os quais destacam-se, neste trabalho, as sincronizações no tempo e na frequência. A primeira está relacionada à identificação do início do primeiro símbolo recebido, enquanto a segunda está associada à correção do erro de frequência de portadora, resultante da diferença entre clocks dos mixers do transmissor e do receptor. Para os esquemas OFDM, Schmidl \& Cox propuseram técnicas para resolver os desafios de sincronismo supracitados com base na transmissão de um símbolo com duas metades idênticas no domínio do tempo [3]. No domínio

O presente trabalho foi realizado com apoio da Coordenação de Aperfeiçoamento de Pessoal de Nível Superior (CAPES) - Código de financiamento 001, do Ministério da Educação (MEC), do Conselho Nacional de Desenvolvimento Científico e Tecnológico (CNPq), do Instituto Nacional de Energia Elétrica (INERGE) e da Fundação de Amparo à Pesquisa do Estado de Minas Gerais (FAPEMIG).

Vanderlan J. E. de Lima, Mateus de L. Filomeno, Ândrei Camponogara e Moisés V. Ribeiro; Universidade Federal de Juiz de Fora (UFJF); endereços de e-mail: \{vanderlan.esterque, mateus.lima, andrei.camponogara, mribeiro\}@engenharia.ufjf.br. da frequência, essa redundância pode ser atingida através da inserção de zeros entre os símbolos modulados [3]. Porém, o mesmo não ocorre quando se trata do domínio de Fresnel. Nesse contexto, este trabalho tem como objetivo encontrar um símbolo no domínio de Fresnel que apresente duas metades idênticas no domínio do tempo e, consequentemente, aplicar a técnica de sincronismo proposta por Schmidl \& Cox [3] em esquemas OCDM.

\section{Modelo do Sistema}

Seja $\mathbf{X}_{i}$ o $i$-ésimo símbolo OCDM de comprimento $N$, no domínio de Fresnel, cujos elementos são obtidos de um modulador digital tal que $\mathbf{X}_{i} \in \mathbb{C}^{N \times 1}$ para sistemas de comunicação operando em banda passante e $\mathbf{X}_{i} \in \mathbb{R}^{N \times 1}$ para banda base. No domínio do tempo discreto, tem-se

$$
\mathbf{x}_{i}=\boldsymbol{\Phi}^{\dagger} \mathbf{X}_{i}
$$

em que $\boldsymbol{\Phi}$ representa a matriz da transformada discreta de Fresnel de tamanho $N$ e $\dagger$ é o operador conjugado transposto.

De acordo com [1], [2], $\boldsymbol{\Phi}=\mathbf{F}_{N}^{\dagger} \boldsymbol{\Gamma} \mathbf{F}_{N}$, em que $\mathbf{F}_{N}$ é a versão normalizada da matriz da transformada discreta de Fourier de tamanho $N$ e $\boldsymbol{\Gamma}=\operatorname{diag}\left\{\Gamma_{0}, \Gamma_{1}, \ldots, \Gamma_{N-1}\right\}$ é a matriz diagonal dos autovalores de $\boldsymbol{\Phi}$. Tais autovalores são definidos para sistemas de comunicação operando em banda passante e em banda base, respectivamente, pelas equações

$$
\Gamma_{k} \triangleq e^{-(j \pi / N) k^{2}}, \forall k \in\{0,1, \ldots, N-1\}
$$

$$
\Gamma_{k} \triangleq \begin{cases}e^{-(j \pi / N) k^{2}}, & \text { para } k<N / 2 \\ e^{(j \pi / N) k^{2}}, & \text { para } k \geq N / 2\end{cases}
$$

Destaca-se que $\boldsymbol{\Phi} \in \mathbb{C}^{N \times N}$ para $\boldsymbol{\Gamma}$ proveniente de (2), enquanto $\boldsymbol{\Phi} \in \mathbb{R}^{N \times N}$ para $\boldsymbol{\Gamma}$ oriundo de (3).

Resumidamente, o símbolo no domínio do tempo discreto, $\mathbf{x}_{i}$, tem ainda a inserção do prefixo cíclico de comprimento $L_{\mathrm{CP}}$ e passa por um conversor paralelo-serial antes de ser convertido a analógico. O sinal resultante é então transmitido através de um canal de comunicação dispersivo de comprimento $L_{h}$, tal que $L_{\mathrm{CP}} \geq L_{h}-1$, e sofre o efeito de um ruído aditivo ao chegar no receptor.

No receptor, por sua vez, as operações inversas àquelas realizadas no transmissor são executadas antes da decodificação dos símbolos recebidos. Porém, para que os símbolos recebidos sejam identificados e corretamente decodificados, faz-se necessária a utilização de técnicas de sincronismo de símbolos no tempo e a correção do erro da frequência de portadora (para sistemas operando na banda passante). 


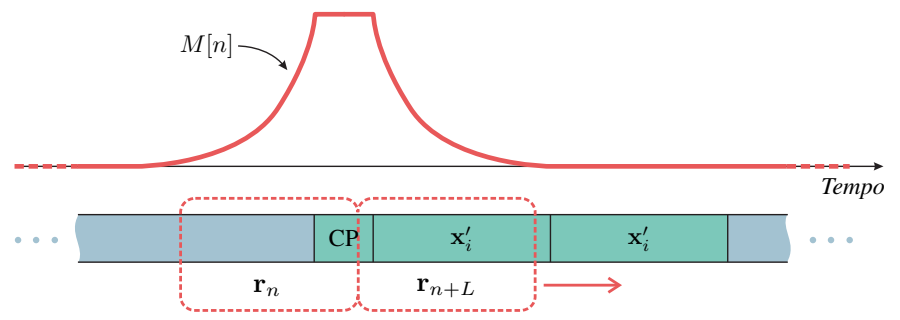

Fig. 1. Sequência da função de correlação normalizada, $\{M[n]\}$, para um símbolo de sincronismo.

\section{SCHMIDL \& COX}

Inicialmente proposta para esquemas OFDM, a técnica de sincronização de $S c h m i d l$ \& Cox [3] é uma técnica robusta que permite tanto a identificação do ponto inicial dos símbolos no domínio do tempo discreto como a correção do erro de frequência de portadora. Esta técnica baseia-se na redundância existente entre duas metades iguais de um mesmo símbolo no domínio do tempo discreto, como explicado a seguir.

\section{A. Sincronização Temporal}

Para a estimação do ponto inicial dos símbolos no domínio do tempo, considera-se símbolos OFDM ou OCDM distintos de seus símbolos anteriores e posteriores, como apresentado na Fig. 1. Note que cada símbolo deve possuir duas metades iguais no domínio do tempo, ou seja, $\mathbf{x}_{i}=\left[\begin{array}{ll}\mathbf{x}_{i}^{\prime T} & \mathbf{x}_{i}^{\prime T}\end{array}\right]^{T}$. Dessa forma, a técnica proposta em [3] pode ser aplicada. Ela baseia-se na correlação entre dois vetores sucessivos de comprimento $L=N / 2$. Observe que a Fig. 1 ilustra o funcionamento dessa técnica, em que os dois vetores deslizam ao longo do sinal coletado pelo receptor. A sequência da função de correlação entre os vetores, normalizada pela energia do segundo vetor, pode ser expressa por

$$
M[n]=\frac{\mathbf{r}_{n}^{\dagger} \mathbf{r}_{n+L}}{\mathbf{r}_{n+L}^{\dagger} \mathbf{r}_{n+L}},
$$

em que $\mathbf{r}_{n}=[r[n] r[n+1] \ldots r[n+L]]^{T}$ e $\{r[n]\}$ é a sequência de símbolos recebidos. De acordo com a Fig. 1, o ponto inicial de cada símbolo encontra-se no platô da sequência $\{M[n]\}$. Dessa forma, uma boa estimativa seria considerar o ponto médio dos platô. No caso de múltiplas estimativas, uma média entre os pontos de sincronismo de cada platô seria efetiva para a redução do efeito do ruído aditivo.

\section{B. Sincronização na Frequência}

Uma vez definido o ponto inicial dos símbolos no domínio do tempo, o erro de frequência de portadora pode ser estimado através da multiplicação do conjugado da primeira metade do símbolo pela segunda metade. Como os duas partes são iguais, tanto a fase do sinal como a fase do canal serão anuladas, restando apenas uma componente de fase proporcional ao erro de frequência de portadora. Dessa forma é possível estimar esse erro e posteriormente cancelar o seu efeito [4].

\section{Símbolo de PreÂmbulo PARA Sincronismo Em ESQUEMAS OCDM}

Nesta seção, visa-se gerar um símbolo OCDM que permita a utilização da técnica de sincronização de Schimdl \& Cox. Uma alternativa seria utilizar uma transformada discreta de Fresnel com metade do comprimento e duplicar o símbolo obtido. Porém, a fim de reduzir a complexidade computacional na etapa de transmissão, busca-se um símbolo OCDM no domínio de Fresnel que possua duas metades iguais no domínio do tempo. Nesse sentido, seja $\mathbf{x}=\left[\mathbf{x}^{\prime T} \mathbf{x}^{\prime T}\right]^{T}$ o símbolo no domínio do tempo com duas metades iguais, $\mathbf{x}^{\prime}$, utilizado para fins de sincronismo. No domínio de Fresnel, tal símbolo é dado por

$$
\mathbf{X}=\mathbf{\Phi} \mathbf{x}=\boldsymbol{\Phi}\left[\mathbf{x}^{\prime T} \mathbf{x}^{\prime T}\right]^{T} .
$$

Observe que a matriz da transformada discreta de Fresnel é quadrada e com dimensões pares; portanto, $\boldsymbol{\Phi}$ pode ser divida em blocos de matrizes de dimensões $N / 2 \times N / 2$ da seguinte maneira:

$$
\boldsymbol{\Phi}=\left[\begin{array}{ll}
\boldsymbol{\Phi}_{11} & \boldsymbol{\Phi}_{12} \\
\boldsymbol{\Phi}_{21} & \boldsymbol{\Phi}_{22}
\end{array}\right] .
$$

Uma vez que a matriz $\boldsymbol{\Phi}$ pode ser decomposta em uma matriz diagonal pré e pós multiplicada pelas versões inversa e direta da matriz da transformada discreta de Fourier, $\boldsymbol{\Phi}$ é uma matriz circulante e, analisando as matrizes $\boldsymbol{\Phi}_{11}, \boldsymbol{\Phi}_{12}, \boldsymbol{\Phi}_{21}$ e $\boldsymbol{\Phi}_{22}$ formadas, nota-se que $\boldsymbol{\Phi}_{11}=\boldsymbol{\Phi}_{22}$ e $\boldsymbol{\Phi}_{12}=\boldsymbol{\Phi}_{21}$. Dessa maneira, (5) pode ser reescrita da seguinte forma:

$$
\mathbf{X}=\left[\begin{array}{ll}
\boldsymbol{\Phi}_{11} & \boldsymbol{\Phi}_{12} \\
\boldsymbol{\Phi}_{12} & \boldsymbol{\Phi}_{11}
\end{array}\right]\left[\begin{array}{l}
\mathbf{x}^{\prime} \\
\mathbf{x}^{\prime}
\end{array}\right]=\left[\begin{array}{l}
\boldsymbol{\Phi}_{11} \mathbf{x}^{\prime}+\boldsymbol{\Phi}_{12} \mathbf{x}^{\prime} \\
\boldsymbol{\Phi}_{11} \mathbf{x}^{\prime}+\boldsymbol{\Phi}_{12} \mathbf{x}^{\prime}
\end{array}\right]
$$

Portanto, para que o símbolo no domínio do tempo seja composto por duas metades iguais, é necessário que o mesmo símbolo no domínio de Fresnel também possua duas metades iguais.

\section{CONSIDERAÇÕES FInAIS}

Neste trabalho foi apresentada uma maneira de se obter um símbolo no domínio de Fresnel cuja representação no domínio do tempo é composta por duas metades idênticas. Baseado na característica circulante da matriz da transformada discreta de Fresnel, foi demonstrado que um símbolo com duas metades iguais no domínio de Fresnel resulta em um símbolo com duas metades iguais no domínio do tempo. Como resultado, a técnica de sincronismo inicialmente proposta para esquemas OFDM por Schmidl \& Cox [3] pode ser aplicada em esquemas OCDM.

\section{REFERÊNCIAS}

[1] X. Ouyang and J. Zhao, "Orthogonal chirp division multiplexing," IEEE Transactions on Communications, vol. 64, no. 9, pp. 3946-3957, 2016.

[2] L. de M. B. A. Dib, G. R. Colen, M. de L. Filomeno, and M. V Ribeiro, "Orthogonal chirp division multiplexing for baseband data communication systems," IEEE Systems Journal, vol. 14, no. 2, pp. 2164-2174, 2020.

[3] T. Schmidl and D. Cox, "Robust frequency and timing synchronization for OFDM," IEEE Transactions on Communications, vol. 45, no. 12, pp. 1613-1621, 1997

[4] T. R. Oliveira, C. A. G. Marques, W. A. Finamore, S. L. Netto, and M. V. Ribeiro, "A methodology for estimating frequency responses of electric power grids," Journal of Control, Automation and Electrical Systems, no. 25, pp. 720-731, 2014. 